\title{
Recovered microbiome of an oviparous lizard differs across gut and reproductive tissues, cloacal swabs, and feces
}

\author{
Marie Bunker ${ }^{1}$, Mark Martin ${ }^{1}$, and Stacey Weiss ${ }^{1}$ \\ ${ }^{1}$ University of Puget Sound
}

June 7,2021

\begin{abstract}
Microbial diversity and community function are related, and can be highly specialized in different gut regions. The cloacal microbiome of Sceloporus virgatus provides antifungal protection to eggshells during oviposition - a specialized function that suggests a specialized microbial composition. Here, we describe the $S$. virgatus cloacal microbiome from tissue and swab samples, and compare it to tissue samples from the gastrointestinal (GI) tract and oviduct, adding to the growing body of evidence of microbiome localization in reptiles. We further assessed whether common methods of microbial sampling - cloacal swabs and feces - provide accurate representations of these microbial communities and whether feces might "seed" the cloacal microbiome or impact the accuracy of cloacal swab sampling. We found that different regions of the gut had unique microbial community structures. The cloacal community, in particular, showed extreme specialization averaging 99\% Proteobacteria (Phylum) and 83\% Enterobacteriacaea (Family). Cloacal swabs recovered communities similar to that of lower intestine and cloacal tissues, but fecal samples had much higher diversity and a distinct composition (62\% Firmicutes and 39\% Lachnospiraceae) relative to all gut regions. Finally, we found that feces and cloacal swabs recover different communities, but cloacal swabs may be contaminated with fecal matter if taken immediately after defecation. These results serve as a caution against the assumption that fecal samples provide an accurate representation of the gut, and that although cloacal swabs can reflect a portion of the lower GI tract microbiome, they may also result in a mixed community of gut and fecal microbes.
\end{abstract}

\section{Introduction}

As high-throughput sequencing becomes more accessible, microbiome studies are expanding beyond humans and mammalian model systems to non-model organisms. It has become clear that the community of microbes within the gut of all animals plays an important role not only in host physiology but also their ecology and evolution (Archie \& Theis, 2011; McFall-Ngai et al., 2013; Moran et al., 2019; Reese \& Dunn, 2018). To better understand the wide-ranging implications of host-microbe co-evolution and the variety of functions the gut microbiome can provide for the host, it is critical to study a broad diversity of host species with a broad diversity of environmental pressures and adaptations. Conducting such research on wild populations is particularly important because captivity has been shown to change the composition of gut microbiomes (Hird, 2017; Keenan et al., 2013; Kohl et al., 2017).

The function and the composition of the gut microbiome can be highly specialized along different regions of the gastrointestinal (GI) tract, which vary in acidity, specific macromolecules secreted by gut epithelia, oxygen content, and other conditions that affect which microbes can thrive (Beasley et al., 2015; Reese \& Dunn, 2018). Regional localization of the gut microbiome is well established in both model and non-model systems (Beasley et al., 2015; Colston et al., 2015; Kohl et al., 2017; Shterzer et al., 2020; Videvall et al., 2018; Yasuda et al., 2015). Among oviparous vertebrates, the terminus of the GI tract is shared with the reproductive tract, as the cloaca is the site of defecation, copulatory intromission, and oviposition. Thus, the cloacal microbiome of birds and reptiles likely experiences unique selective pressures relative to the rectal microbiome of mammals. For instance, the cloacal microbiome may be affected by breeding season, sex, 
degree of promiscuity, and other aspects of host reproduction and behavior (Escallón et al., 2019; Lee, 2015; White et al., 2011).

In the striped plateau lizard, Sceloporus virgatus, free-ranging females lay their eggs in soil burrows at the onset of the summer monsoon rains and then leave, providing no further parental care (Rose, 1981). Timing oviposition during the monsoon season appears to be unique to $S$. virgatus among temperate lizard species, and has been hypothesized to have a large selective impact on the species (Vinegar, 1975). We propose a strong selective impact on the species' microbiome as well, resulting in a cloacal microbiome that protects host's eggs from fungal pathogens that thrive during the wet monsoon period (Bunker et al., in review). Indeed, microbes are transferred from the cloacae of $S$. virgatus females to eggshells during egg laying, reducing fungal attachment to eggs and improving hatch success (Bunker et al., in review). Given this specialized ecological function, we further hypothesized that the gut microbiome of $S$. virgatus females will express a high degree of localization along the GI and reproductive tracts. While such localization has been found in several other reptile species (Colston et al., 2015; Costello et al., 2010; Keenan et al., 2013; Kohl et al., 2017), we predicted a pattern in contrast to that found in these other reptiles; specifically, we predicted that the cloaca will show reduced microbial diversity and a unique community structure relative to upper regions. To test this, we compared the microbiome of the cloaca, lower and upper intestinal regions, and the oviduct. Because previous work on the S. virgatus microbiome has been based on cloacal swab samples (Bunker et al., in review; Martin et al., 2010), we additionally assessed whether the cloacal microbiome determined from cloacal swabs was representative of cloacal tissue or other regions of the GI and reproductive tracts. We predicted that the less invasive swab samples would serve as a good proxy for the more invasive tissue sampling of the cloaca, but would be less suitable as a proxy of upper GI and reproductive tract tissues.

It is common among studies of reptiles and birds for cloacal swabs and/or fecal samples to be used as a proxy for the gut microbiome as a whole (Colston et al., 2015; Escallón et al., 2019; Hong et al., 2011; Jiang et al., 2017; Kohl et al., 2017; Kreisinger et al., 2015). These methods are attractive as they are non-destructive, minimally invasive, and allow for repeat sampling of the same individuals and communities over time (Berlow et al., 2020; Videvall et al., 2018). However, a microbial community recovered from swabs or feces may represent only a portion of the gut due to the regional localization. While only a few studies have directly compared the two non-invasive sampling approaches, unique community structures have been recovered by cloacal swab and fecal sampling in zebra finches and ostriches (Berlow et al., 2020; Videvall et al., 2018). Here, we add to this important work by also directly comparing microbial communities collected from cloacal swab and fecal samples of $S$. virgatus individuals. If the communities are similar, feces may inoculate the cloaca with microbes as they pass through it. However, we predicted that the communities will be distinct in $S$. virgatus, indicating selection for the particular cohort of microbes that occupy the cloaca, due in part to the natural history of $S$. virgatus (as detailed above). If indeed the cloacal and fecal microbial communities are distinct, it is possible that cloacal swab samples can be contaminated by small particles of fecal material that remain in the cloaca following defecation and then attach to the swab. Unintended fecal contamination of swab samples may or may not be visible on the swab but could potentially help explain the high variation recovered from such samples in other studies (Videvall et al., 2018; Williams \& Athrey, 2020; Bunker and Weiss, unpublished data). To test these ideas, we compared microbial communities detected from cloacal swabs collected before and after defecation. If contamination was occurring, we predicted that communities recovered from cloacal swabs immediately after defecation would have higher diversity and a different community structure than swabs taken prior to defecation.

\section{Methods}

Study 1: Is the cloaca a uniquely specialized region of the gastrointestinal and reproductive tracts?

Sceloporus virgatus lizards are small insectivorous animals found in the Madrean Sky Islands of the southwestern United States and south into Mexico (Stebbins, 2003). Our study population is at the northern reach of their range, in Cochise County, Arizona near the American Museum of Natural History's Southwestern Research Station (SWRS). To address the adaptive hypothesis that the cloacal microbiome will be unique from that of the upper GI and reproductive tracts, we collected gravid Sceloporus virgatusfemales $(\mathrm{n}=8)$ 
between 28 June and 01 July 2019 using a loop of fishing line tied to a retractable fishing pole. Female cloacae were swabbed immediately following capture. Females were then kept in large outdoor enclosures at SWRS with access to water bowls and naturally occurring prey for 1-4 d until being shipped overnight to the University of Puget Sound in individual plastic containers on ice packs. Upon arrival, we euthanized females using buffered MS-222 (Conroy et al., 2009). We collected transverse sections of tissue $(\sim 2-4 \mathrm{~mm}$ long) from the cloaca (expanded tissue immediately above the vent), lower intestine ( $\sim 10 \mathrm{~mm}$ above cloaca), upper intestine ( $\sim 5 \mathrm{~mm}$ below stomach), and oviduct (at the position of the lowest egg on the left side) using heat sterilized instruments, and did not attempt to separate or isolate mucosal vs. luminal contents. Microbes were eluted from swabs into an Amies solution by manual shaking and were stored at $-80^{\circ} \mathrm{C}$, as were tissue samples, until DNA extraction.

Study 2: How does sampling methodology (swab vs feces) affect recovered community composition?

To assess the efficacy of cloacal swab and fecal sampling in $S$. virgatus, we collected 8 adult lizards $(\mathrm{n}=4$ females and $\mathrm{n}=4$ males) on 30 May 2019 using the same sites and methodology described above. Animals were housed individually in sterile $15 \times 23 \mathrm{~cm}$ plastic tanks lined with paper towels that had also been sterilized with $70 \%$ ethanol on a west-facing screened porch of SWRS's Live Animal Holding Facility. They were offered a single cricket on the day of capture that was removed if not eaten by $\sim 1200$ the next day. Sterile water was provided ad libitum, and one heat lamp on a 14:10 light cycle was shared between two adjacent tanks.

Lizard cloacae were swabbed first at $\sim 0500$ on 31 May, before animals had woken up and defecated; this is considered our pre-defecation swab sample. Beginning at 0600, the tanks were checked for feces every 30 min, until 1830, when the heat lamps had been off for $30 \mathrm{~min}$ and the lizards were no longer active. When a fecal pellet was found, it was collected with sterile tweezers and the lizard's cloaca was immediately swabbed. Based on this method, all post-defecation swabs were taken within $30 \mathrm{~min}$ of the defecation event. We also collected a control swab, which we used to sample the researchers' hands, a lizard's external vent and belly, and the air of the porch, to collect any microbes that may have contaminated cloacal swabs during sampling.

\section{DNA Extraction}

DNA from tissue, swab, and fecal samples were extracted using the Qiagen DNEasy Blood and Tissue Kit (Qiagen, Inc). For the cloacal swab samples, we used the manufacturer protocol for Purification of Total DNA from Animal Blood or Cells with the optional pre-treatment for Gram-positive bacteria lysis buffer incubation. For tissue and fecal pellets, we included the optional lysis buffer incubation for $30 \mathrm{~min}$ at $37 \mathrm{degC}$. After incubation, Buffer AL and proteinase $\mathrm{K}$ were added to the tubes. The samples were then incubated at $56 \mathrm{deg}$ C for 180 minutes (for tissue) or $90 \mathrm{~min}$ (for feces) while shaking at 500 RPM (USA Scientific Mixer HC- 8012-0000, Ocala, FL, USA). From here, the extraction was completed according to the Purification of Total DNA from Animal Blood or Cells protocol, beginning at Step 3 (addition of pure ethanol). An extraction blank was included in each extraction. DNA in all samples and blanks was quantified via Qubit to ensure extraction was successful.

\section{Illumina Library Prep}

We used a two-step polymerase chain reaction (PCR) procedure modified from the IBest Genomics Core at University of Idaho to amplify the 16s V4 region of the microbial DNA in each sample (Taylor et al., 2019). PCR1 utilized 515F/806R primer pairs to amplify the region of interest, and PCR2 extended the amplicons with sample-specific barcodes. For PCR1, we used 7.5 uL Phusion Flash High Fidelity Master Mix (ThermoFisher, Waltham, MA), $0.15 \mathrm{uL}$ of the forward and reverse $50 \mu \mathrm{M}$ primers, $0.75 \mathrm{uL}$ molecular grade Bovine Serum Albumen (BSA, $20 \mathrm{mg} / \mathrm{mL}$, New England BioLabs), $5.45 \mathrm{uL}$ purified water, and 1 $\mathrm{uL}$ template DNA. The thermal cycling protocol was: initial denaturation at $98^{\circ} \mathrm{C}$ for $10 \mathrm{sec}$; 28 cycles of denaturation at $98^{\circ} \mathrm{C}$ for $1 \mathrm{sec}$, annealing at $57^{\circ} \mathrm{C}$ for $5 \mathrm{sec}$, and extension at $72^{\circ} \mathrm{C}$ for $20 \mathrm{sec}$, with a final extension at $72^{\circ} \mathrm{C}$ for $60 \mathrm{sec}$ (Taylor et al., 2019). We visualized PCR products on a $2 \%$ agarose gel using 10x Sybr Green (Molecular Probes, Invitrogen; Carlsbad, CA). All iterations of PCR1 included a positive (Serratia genomic DNA) and a negative (purified water) control. Mock communities (BEI Resources, ATCC, 
Manassas, VA) were also included in some PCR1 runs, as a further measure of quality control.

We performed PCR1 in triplicate for each sample, then pooled those replicates for PCR2. Samples (including positive controls) that showed strong bands in at least two of three PCR1 replicates were diluted 1:4 in purified water before being used as template DNA in PCR2, and all others were undiluted. In addition to pooling replicates of the negative controls, negative controls from different PCR1 runs were further pooled to minimize the number of samples sent for sequencing. Because of this, some iterations of PCR2 contained a pooled PCR1 negative control, and some contained a new PCR2 negative control with purified water instead of DNA template.

For PCR2, we used $10 \mathrm{uL}$ Phusion Flash High Fidelity Master Mix, $0.24 \mathrm{uL}$ BSA, $8.01 \mathrm{uL}$ of purified water, $0.75 \mathrm{uL}$ of unique barcoded primer pairs $(2 \mu \mathrm{M}$; supplied by IBest Genomics Core, at the University of Idaho; see Taylor et al., 2019), and 1 uL of the PCR1 pooled product. The thermal cycling protocol was the same as the protocol for PCR1, but was run for only 8 cycles (for 36 cycles total). We visualized PCR2 products on a $2 \%$ agarose gel with 10x Sybr Green and confirmed that each sample had undergone a band shift compared to PCR1 (indicative of attachment of barcode primers). We then pooled all samples, with pool volume based on the intensity of each sample band, and shipped them to IBest Genomic Core for purification, DNA quantification, and sequencing on the Illumina MiSeq platform. All negative controls and mock communities were included in the sequencing process, but positive controls were not.

\section{Illumina Raw Data Processing and Statistical Analysis}

Sequences were received demultiplexed, with adapters and primers removed. Quality analysis for each sample was performed using FastQC (Andrews, 2010) and those results were consolidated using MulitQC (Ewels et al., 2016). Mean quality scores and length distribution for the whole dataset were manually inspected and used to determine a cutoff length of $250 \mathrm{bp}$ for forward reads and $150 \mathrm{bp}$ for reverse reads. Samples were then processed in R v3.1.6 (R Core Team, 2020) using the DADA2 (Callahan et al., 2016) pipeline based on this tutorial:https://benjjneb.github.io/dada2/tutorial.html. Samples were trimmed as described above and filtered with a max expected error of 2 . An average of $91.6 \%$ of reads were kept in all experimental samples after processing. Taxonomic classification of amplified sequence variants (ASVs) was performed through the assignTaxonomy function, using the Silva database (Quast et al., 2013), release 132. Potential contaminants were removed with the Decontam package (Davis et al., 2018), using the "prevalence" method with a threshold of 0.1. Control samples, including a control swab $(\mathrm{n}=1)$, extraction blanks $(\mathrm{n}=9)$, and PCR negatives $(\mathrm{n}=24)$, were used for comparison. Any ASV that had fewer than 27 reads across all samples was discarded. One cloacal tissue sample only kept 12 reads after processing and was not included in analyses. Finally, read numbers were log transformed to account for differences in read depth. All parameters were determined based on an analysis of the mock communities.

Once samples had been processed, the phyloseq package (McMurdie \& Holmes, 2013) was used to organize and store data of different types for analyses. Shannon diversity index values and richness were then calculated with the "estimate_richness_ function" from phyloseq, and Faith's phylogenetic diversity index values were calculated using the Picante package (Kembel et al., 2010) based on phylogenetic trees created and optimized with Phangorn (Callahan et al., 2016; Schliep et al., 2016). The alignment was created with the DECIPHER package (Wright, 2016). We examined how these variables differed across tissue types and swab samples using mixed-effects ANOVAs with lizard ID as a random effect. We compared fecal and predefecation swab communities, as well as pre- and post-defecation swab communities using separate paired t-tests. Richness was log-transformed to account for non-normal distribution when comparing pre- and post-defecation swabs. Phylogenetic diversity was log transformed when comparing tissue types.

Pairwise distances between samples were calculated by the vegan package (Oksanen et al., 2019) using Bray-Curtis distances, and these distances were then used to generate non-metric multidimensional scaling (NMDS) plots. Using the trees described above, we used phyloseq to generate weighted UniFrac distances to compare phylogenetic community composition, and we used unweighted UniFrac distance to compare community membership of groups; both metrics were used to generate Principle Coordinate Analysis (PCoA) 
plots. For all distance metrics, dispersion between groups was first tested with the betadisper function from the vegan package, and then a PERMANOVA test (Adonis function from the vegan package) was performed. Differential abundance was tested using the Corncob package (Martin et al., 2020). All plots were made with the GGplot2 package (Wickham, 2017).

\section{Results}

Study 1: Is the cloaca a uniquely specialized region of the gastrointestinal and reproductive tracts?

The Shannon diversity value was lowest in the cloacal tissue, on average, but was not statistically different across tissue samples and cloacal swabs $(\mathrm{F}=1.32 \mathrm{df}=4,26, \mathrm{p}=0.425$, Fig 1a). Similar non-significant patterns were found when comparing richness $(\mathrm{F}=2.07, \mathrm{df}=4,26, \mathrm{p}=0.114$; Fig $1 \mathrm{~b})$ and phylogenetic diversity across tissue types $(\mathrm{F}=1.30 \mathrm{df}=4,26, \mathrm{p}=0.297$; Fig $1 \mathrm{c})$. When comparing beta diversity, measured by Bray-Curtis distance, there was a significant difference in composition between all groups $(\mathrm{F}=$ $1.52, \mathrm{df}=4,33, \mathrm{p}=0.014)$, although the groups were dispersed similarly $(\mathrm{F}=1.04, \mathrm{df}=4,33, \mathrm{p}=0.400$, Fig 2a). When clustered using weighted UniFrac distances, community composition tended to differ across all tissue types $(\mathrm{F}=1.87, \mathrm{df}=4,33, \mathrm{p}=0.060$, Fig $2 \mathrm{~b})$, although the groups were dispersed differently $(\mathrm{F}$ $=4.12, \mathrm{df}=4,33, \mathrm{p}=0.008)$, which could account for the difference in distances. When clustered using unweighted UniFrac distances, community membership differed significantly between tissue types $(\mathrm{F}=1.66$, $\mathrm{df}=4,33, \mathrm{p}=0.008, \mathrm{Fig} 2 \mathrm{c})$, and the tissue types showed similar dispersion $(\mathrm{F}=1.16, \mathrm{df}=4,33, \mathrm{p}=$ $0.346)$.

The most abundant family in all tissues types wasEnterobacteriaceae (Figure 3; Online Resource 1). In the cloacal tissue, on average, Enterobacteriaceae made up $82.7 \pm 10.8 \%$ (SE) of the whole community, while the next most abundant family (Helicobacteraceae) only made up $16.6 \pm 10.9 \%$ and was $>0.1 \%$ in only 2 individuals. No other families made up more than $1 \%$ of the composition of the cloacal tissue. The cloacal swabs and lower intestine showed similar patterns:Enterobacteriaceae made up $69.8 \pm 14.2 \%$ and $64.7 \pm$ $11.5 \%$ respectively, and the next most abundant family in both wasHelicobacteraceae. However, both the cloacal swabs and the lower intestine had several families that made up between 1-5\% of the community, including Bacteroidaceae, Ruminococcaceae, Tannerellaceae, Lachnospiraceae, andDesulfovibrionaceae . The upper intestine had the lowest percent composition of Enterobacteriaceae with only $34.9 \pm 9.1 \%$, closely followed by Ruminococcaceae which made up $32.9 \pm 12.8 \%$ on average, and both Bacteroidaceae and Burkholderiaceae made up $\sim 9 \%( \pm 7.5, \pm 9.0)$. The oviduct community was $54.9 \pm 9.3 \%$ Enterobacteriaceae on average and had many families that were between $1-5 \%$, but no others higher than $10 \%$.

A differential abundance analysis showed that the percentage of Enterobacteriaceae found in the cloacal tissue microbial community was similar to that in the cloacal swabs $(\mathrm{t}=-1.05 \mathrm{p}=0.304)$ and lower intestine $(\mathrm{t}$ $=0.156, \mathrm{p}=0.372)$ communities, and was significantly greater than that in the upper intestine $(\mathrm{t}=-3.09$, $\mathrm{p}=0.005)$ and oviduct $(\mathrm{t}=-2.06, \mathrm{p}=0.049)$ communities (Online Resource 1 ). It also showed that the cloacal tissue community had distinctly low percentages of Ruminococcaceae (cloacal swab: $\mathrm{t}=4.39, \mathrm{p}<$ 0.001; lower intestine: $\mathrm{t}=4.38, \mathrm{p}<0.001$; upper intestine: $\mathrm{t}=7.94, \mathrm{p}<<0.001$; oviduct: $\mathrm{t}=2.49, \mathrm{p}=$ 0.019 ) and Bacteroidaceae (cloacal swab: $\mathrm{t}=4.88, \mathrm{p}<0.001$; lower intestine: $\mathrm{t}=4.88, \mathrm{p}<0.001$; upper intestine: $\mathrm{t}=5.07, \mathrm{p}<<0.001$; oviduct: $\mathrm{t}=3.59, \mathrm{p}=0.001$ ).

Study 2: How does sampling methodology (swab vs feces) affect recovered community composition?

Shannon diversity index values were significantly higher in fecal pellets than in pre-defecation cloacal swabs $(\mathrm{t}=16.29, \mathrm{df}=7, \mathrm{p}<0.001 ;$ Fig $4 \mathrm{a})$, as was richness $(\mathrm{t}=8.39, \mathrm{df}=, 7, \mathrm{p}<0.001 ;$ Fig $4 \mathrm{~b})$ and phylogenetic diversity $(\mathrm{F}=10.94$, df $=7, \mathrm{p}<0.001$; Fig $4 \mathrm{c}$ ). Beta diversity analysis using Bray-Curtis distance showed that the microbial composition of fecal pellets was significantly more similar to other fecal pellets than it was to that of the pre-defecation cloacal swabs $(\mathrm{F}=9.65 \mathrm{df}=1,14, \mathrm{p}=0.002$; Fig $5 \mathrm{a})$. The groups were also dispersed differently $(\mathrm{F}=5.54 \mathrm{df}=1,14, \mathrm{p}=0.034)$. Fecal pellets also differed from pre-defecation cloacal swabs in both UniFrac metrics (weighted: $\mathrm{F}=18.91$, $\mathrm{df}=1,14, \mathrm{p}=0.001$, Fig $5 \mathrm{~b}$; unweighted: $\mathrm{F}=$ 15.13 , df $=1,14, \mathrm{p}=0.001$, Fig $5 \mathrm{c}$ ), and dispersion of the communities also varied for each measurement (weighted: $\mathrm{F}=13.83, \mathrm{df}=1,14, \mathrm{p}=0.002$; unweighted: $\mathrm{F}=4.78, \mathrm{df}=1,14, \mathrm{p}=0.046$ ). 
There was no significant difference between pre- and post-defecation swabs in any alpha diversity metric (Shannon: $\mathrm{t}=1.40, \mathrm{df}=7, \mathrm{p}=0.204$; Richness: $\mathrm{t}=1.45, \mathrm{df}=7, \mathrm{p}=0.190$; Phylogenetic diversity: $\mathrm{t}=$ 1.72 , df $=7, \mathrm{p}=0.129$; Fig 4), although the post-defecation swab tended to have higher diversity. Swab types did not cluster separately based on Bray-Curtis distances $(\mathrm{F}=1.24$, df $=1,14, \mathrm{p}=0.231$, Fig 5a), and the groups were dispersed similarly $(\mathrm{F}=2.57 \mathrm{df}=1,14, \mathrm{p}=0.131)$. Pre-defecation swab communities differed from post-defecation swab communities in dispersion based on weighted $(\mathrm{F}=7.08, \mathrm{df}=1,14, \mathrm{p}$ $=0.019$, Fig $5 \mathrm{~b})$ and unweighted $(\mathrm{F}=36.14, \mathrm{df}=1,14, \mathrm{p}<0.001$, Fig $5 \mathrm{c})$ UniFrac distances, as well as composition of unweighted UniFrac distance $(\mathrm{F}=2.20 \mathrm{df}=1,14, \mathrm{p}=0.035)$, although this could be due to differences in dispersion rather than true clustering of groups. The different swab types did not cluster separately based on weighted UniFrac distance $(\mathrm{F}=1.14$, $\mathrm{df}=1,14, \mathrm{p}=0.281)$.

The most abundant taxa recovered from fecal pellets was Lachnospiraceae, which made up $38.7 \pm 5.2 \%$ on average (Fig 6). Enterobacteriaceae, Bacteroidaceae, andRuminococcacea each accounted for $~ 10 \%$ of the recovered communities, and several others made up between 1-5\% of the recovered community including Tannerellaceae, Erysipelotrichaceae, Eggerthellaceae, Marinifilaceae, and Akkermansiaceae.Pre-defecation cloacal swab communities were dominated byEnterobacteriaceae $(84.9 \pm 5.3 \%)$, and the next most abundant taxa was Helicobacteraceae, which only accounted for $5.1 \pm 2.2 \%$ of the community. A corncob analysis confirmed thatLachnospiraceae was significantly more abundant $(\mathrm{t}=-7.20, \mathrm{p}<0.001)$ and Enterobacteriaceae was significantly less abundant $(\mathrm{t}=3.83, \mathrm{p}<0.001)$ in fecal samples compared to pre-defecation cloacal swabs.

While the same two taxa dominated the pre- and post-defecation swabs, post-defecationEnterobacteriaceae was reduced to only $44.8 \pm 13.2 \%$, and Helicobacteraceae increased to $19.0 \pm 9.5 \%$. There was also an increase in the abundance of feces-associated taxa, particularly Lachnospiraceae which accounted for $15 \pm 7.4 \%$ on average. However, there was a distinct bifurcation in the post-defecation swab samples. In five samples, Enterobacteriaceae andHelicobacteraceae account for $>75 \%$ of the community, similar to the pre-defecation swabs, while the remaining three samples had a more "feces-like" community, with a higher abundance of Lachnospiraceae and Bacteroidaceae.

Does the fecal pellet microbiome represent the endogenous microbiome?

Even though fecal pellets and tissues were collected for different studies and from different animals, we compare their microbial communities descriptively. The three most abundant families in fecal pellets (Lachnospiraceae , Ruminococcaceae, andBacteroidaceae ) combined made up an average of $60 \%$ of the fecal microbial community, $43 \%$ of the upper intestine community, and less than $1 \%$ of the cloacal community (Online Resource 1). The similarity between fecal and the upper intestine communities is predominantly due to the high abundance of Ruminococcaceae andBacteroidaceae in the upper intestine. However, despite the high abundance of Lachnospiraceae in fecal pellets, only about $1 \%$ of the upper intestine community was made up of Lachnospiraceae . At the phylum level, fecal pellet communities averaged $62 \%$ Firmicutes, $18 \%$ Bacteroidetes, and 11\% Proteobacteria (Fig 7). As we sampled down the digestive and reproductive tracts, the percentage of Firmicutes and Bacteroidetes steadily decreased and the percentage of Proteobacteria increased, to the point of nearly totally dominating the cloacal community at an average of $99 \%$ Proteobacteria found in cloacal tissues (Fig 7).

\section{Discussion}

We found clear localization of the microbiome in the $S$. virgatusgut and reproductive tracts. While localization has been seen in other reptiles (Colston et al., 2015; Costello et al., 2010; Keenan et al., 2013; Kohl et al., 2017), the cloaca in previously sampled reptiles was found to have relatively high diversity and to host representatives from the upper regions of the gut. This pattern may be expected as the cloaca is the terminus of the GI and reproductive systems, and is often assumed to be both inoculated by feces that pass through and influenced by sexual transmission during copulation (Videvall et al., 2018; Wen et al., 2021; White et al., 2011). Similarly, the reproductive tract of chickens shows increasing levels of diversity from oviducts to cloaca, which has the highest diversity (Wen et al., 2021). In contrast to these previously described patterns, the cloacal microbiome of $S$. virgatus females is low diversity, especially at the Family 
and Phylum level at which it was dominated byEnterobacteriaceae and Protobacteria, and is distinct from upper intestine, oviductal, and fecal microbiomes. This pattern may indicate strong selection for the specific cohort of bacteria found in the S. virgatus cloaca. S. virgatus females deposit beneficial microbes on their eggs during oviposition that facilitate egg survival (Bunker et al., in review). This function could be the driving force behind the winnowing of the microbiome in the cloaca, especially considering that many members of the Enterobacteriaceae family are known to have antifungal properties (Dhar Purkayastha et al., 2018; Gutiérrez-Román et al., 2015; Kalbe et al., 1996).

Cloacal swabs taken from a given individual lizard resulted in communities similar to the lower intestine and the cloacal tissue, and distinct from higher regions of the gut and reproductive tract. The cloacal swab community was predominantly Enterobacteriaceae but, like the lower intestine, had several families that made small but noticeable contributions to the community and was dispersed slightly differently than the cloacal tissue microbiome. The upper intestine and oviduct have different microbial communities than the lower sections of the intestinal tract but also have differences in major families from one another. Recent evidence has contradicted the long-accepted idea that embryonic development is sterile (Funkhouser \& Bordenstein, 2013). Microbial communities similar to gut microbes have been found in chicken oviducts (Shterzer et al., 2020), and Trevaline et al. (2018) found that microbes may colonize bird and lizard eggs from within the oviduct before the shell develops. The unique community in the oviduct here could indicate an internal egg microbiome which is seeded during egg development; the functional significance of this microbiome should be investigated further.

In contrast to the adequate sampling of the cloacal and lower intestinal microbial communities by cloacal swabs, fecal pellets contain strikingly distinct communities from all gut regions, with greatest similarity to the upper intestine. Pre-defecation cloacal swabs and the fecal samples taken from the same individuals were found to have different microbial communities from one another by every metric we examined. As described above, the swabs were more similar to the cloaca and lower intestine, and largely dominated by Enterobacteriaceae. The fecal sample community was most similar to that found in the upper intestine, with a relatively high abundance of Ruminococcaceae and Bacteroidaceae, but was dominated by Lachnospiraceae which was largely absent from all regions of the gut, and lacked the high abundance of Enterobacteriaceae that was found across the gut. Overall, these patterns caution against the common practice of using fecal samples as a proxy for the gut microbiome without first validating this approach.

While Study 2 found some changes between pre- and post-defecation, those changes were largely nonsignificant, and likely due to increased variation within the post-defecation swabs, as indicated by differences in dispersion, more so than composition, between time points. The variation is due to a distinct bifurcation in the recovered swab communities from post-defecation swabs, with most of these swabs mirroring cloacal tissue, with its high abundance of Proteobacteria, and a smaller portion displaying a more "feces-like" community. This variation in post-defecation cloacal swabs was also present in the field swabs of lizards from Study 1; 5 of the 8 cloacal swab communities from Study 1 were made up of $>95 \%$ Enterobacteriaceae andHelicobacteraceae, while other samples were dominated byLachnospiraceae and Bacteroidaceae, two feces-associated taxa. Because recovered communities from feces and pre-defecation cloacal swabs were distinct, it seems unlikely that the cloaca is being inoculated with fecal microbes. Rather, a more likely explanation is that small amounts of fecal material may attach to swabs if they are taken shortly after defecation, which then masks the much less diverse cloacal community when it is sequenced. The idea of temporary contamination is further supported by the fact that the same lizards, when re-swabbed for a different study, often had comparatively lower diversity and a greater relative abundance of Enterobacteriaceae -associated ASVs (Online Resource 3). Some animals continued to vary between the feces-like and cloacal tissue phenotype when they were resampled, likely due to repeated defecation events. Cloacal swabs often have been found to be inconsistent and unreliable (Videvall et al., 2018; Williams \& Athrey, 2020), and potential contamination by fecal material could account for some of this variation. Indeed, cloacal swabs have been used to collect feces in the past (Stanley et al., 2015). The fact that these fecal microbes do not colonize and grow in the $S$. virgatus cloaca supports the hypothesis that the low microbial diversity of the cloaca is due to selection, and that there is a mechanism to maintain stability of the microbiome in that 
region. This type of selection has been seen in other species (Nyholm \& McFall-Ngai, 2004; Zhang et al., 2016), and supports a model in which function is the driving force of microbiome diversity (Reese \& Dunn, 2018).

At the phylum level, Bacteroidetes and Firmicutes are often considered to represent the core gut microbiome across vertebrates (Colston \& Jackson, 2016; Ley et al., 2008). However, this dogma is based largely on studies that rely on fecal sampling in mammals and so may not accurately represent endogenous microbial communities of diverse vertebrate taxa. For instance, Bacteroidetes have been found to be relatively rare in some gut regions of wild reptiles and birds (Colston et al., 2015; Hird, 2017; Keenan et al., 2013; Kreisinger et al., 2015), and were relatively uncommon across all $S$. virgatus gut regions and even in fecal pellets, which were dominated by Firmicutes. Firmicutes and Proteobacteria were more equally represented in the S. virgatusupper intestine, though Proteobacteria were more common, and Proteobacteria increased in dominance down the GI tract to the cloaca, which was nearly entirely composed of this phylum. Proteobacteria have been found to be highly abundant in the GI tract of other non-mammalian vertebrates (Colston \& Jackson, 2016), particularly in studies that sampled directly from the GI tract rather than relying on fecal sampling alone, but its dominance in the $S$. virgatus cloaca appears to be extreme even in comparison to those systems. While other studies have found a majority of the microbiome was composed of Proteobacteria, the lowest relative abundance of this cloacal community member was $96.9 \%$ with greater than $99 \%$ on average, and the majority of those reads belonged to a single family. The cloacal swabs were less consistent, as discussed above, although still $85 \%$ Proteobacteria on average, and seemed to land between the cloacal tissue community and that of the lower intestine.

Variation in the endogenous microbiome across taxa has been considered in relation to diet and other aspects of animal life history and ecology (Colston \& Jackson, 2016; Ley et al., 2008). As descriptions of vertebrate microbiomes continue to accumulate, it will be interesting to examine the potential influence of reproductive mode (i.e., viviparity vs. oviparity), as this separates mammals from most non-mammalian vertebrates. We propose that oviparous species, and especially those without egg-tending, may have unique selective pressures on the cloacal microbiome to transfer antifungal or otherwise egg-protective bacteria to eggshells during oviposition. Future research will compare the cloacal microbiome of oviparous and viviparous Sceloporus lizards.

\section{Conclusions}

The community structure and composition of the microbiome varies depending on tissue type in the gastrointestinal and reproductive tracts of $S$. virgatus. While environmental factors have been known to cause regional variation in the gut microbiome, in this case there is evidence (Bunker et al., in review) that the difference is due to selection for cloacal microbes that increase host fitness, specifically via the transfer of antifungal microbes from mother to eggshells during oviposition. Additionally, although cloacal swabs and fecal samples are generally accepted methods of sampling the microbiome, neither was able to fully represent the entire community of the gut, and the two methods provided unique results. The cloacal swabs adequately sampled communities of cloacal and lower intestine tissues, whereas the fecal community sampled a distinct community not representative of any sampled gut tissue. We also found that fecal microbes do not seed the cloaca after defecation; while there were few differences between swabs taken before and after defecation overall, there was still evidence of possible fecal contamination of individual swabs. Care should be taken to account for this in future research. Studies that intend to use cloacal swabs or feces as a proxy for the gut microbiome must think carefully on whether these methods will accurately answer their question, or whether a specific, region-based study would be more appropriate. In this study, biologically relevant variation in the microbiome could have been masked due to sampling method.

\section{Acknowledgments}

We thank Grace Elliott and Helena Heyer-Gray for their help with animal collection, and Marley Weiss for help with tissue collection. Michal Morrison-Kerr and staff at the Southwestern Research Station provided logistical assistance. Funding was provided by the National Science Foundation (Grant number 1755408). 
The work was permitted by the University of Puget Sound Institutional Animal Care and Use Committee (PS18002) and Arizona Game and Fish collecting permit (SP649069).

\section{References}

Archie, E. A., \& Theis, K. R. (2011). Animal behaviour meets microbial ecology.Animal Behaviour, 82(3), 425-436. https://doi.org/10.1016/j.anbehav.2011.05.029

Beasley, D. E., Koltz, A. M., Lambert, J. E., Fierer, N., \& Dunn, R. R. (2015). The Evolution of stomach acidity and its relevance to the human microbiome. PLOS ONE, 10(7), e0134116. https://doi.org/10.1371/journal.pone.0134116

Berlow, M., Kohl, K. D., \& Derryberry, E. P. (2020). Evaluation of non-lethal gut microbiome sampling methods in a passerine bird. Ibis, 162(3), 911-923. https://doi.org/10.1111/ibi.12807

Bunker, M. E., Elliot, G., Heyer-Grey, H., Martin, M. O., Arnold, A. E., \& Weiss, S. L. (in review). Vertically transmitted microbiome protects eggs from fungal infection and egg failure. BMC Microbiology.

Callahan, B., McMurdie, P., Rosen, M., Han, A., Johnson, A., \& Holmes, S. (2016). DADA2: High resolution sample inference from Illumina amplicon data.Nature Methods, 13(7).

Callahan, B., Sankaran, K., Fukuyama, J. A., McMurdie, P., \& Holmes, S. (2016). Bioconductor Workflow for Microbiome Data Analysis: From raw reads to community analyses. F1000Research, 5, 1492. https://doi.org/10.12688/f1000research.8986.2

Colston, T. J., \& Jackson, C. R. (2016). Microbiome evolution along divergent branches of the vertebrate tree of life: What is known and unknown. Molecular Ecology, 25(16), 3776-3800. https://doi.org/10.1111/mec.13730

Colston, T. J., Noonan, B. P., \& Jackson, C. R. (2015). Phylogenetic analysis of bacterial communities in different regions of the gastrointestinal tract of Agkistrodon piscivorus, the cottonmouth snake. PLOS ONE, 10(6), e0128793. https://doi.org/10.1371/journal.pone.0128793

Conroy, C., Papenfuss, T., Parker, J., \& Hahn, N. (2009). Use of tricaine methanesulfonate (MS222) for euthanasia of reptiles. Journal of the American Association for Laboratory Animal Science : JAALAS, 48(1), 28-32.

Costello, E. K., Gordon, J. I., Secor, S. M., \& Knight, R. (2010). Postprandial remodeling of the gut microbiota in Burmese pythons. The ISME Journal, 4(11), 1375-1385. https://doi.org/10.1038/ismej.2010.71

Davis, N. M., Proctor, D. M., Holmes, S. P., Relman, D. A., \& Callahan, B. J. (2018). Simple statistical identification and removal of contaminant sequences in marker-gene and metagenomics data. Microbiome, 6(1), 226. https://doi.org/10.1186/s40168-018-0605-2

Dhar Purkayastha, G., Mangar, P., Saha, A., \& Saha, D. (2018). Evaluation of the biocontrol efficacy of a Serratia marcescens strain indigenous to tea rhizosphere for the management of root rot disease in tea. PLoS ONE, 13(2). https://doi.org/10.1371/journal.pone.0191761

Escallon, C., Belden, L. K., \& Moore, I. T. (2019). The Cloacal microbiome changes with the breeding season in a wild bird. Integrative Organismal Biology,1(1). https://doi.org/10.1093/iob/oby009

Ewels, P., Magnusson, M., Lundin, S., \& Kaller, M. (2016). MultiQC: Summarize analysis results for multiple tools and samples in a single report.Bioinformatics, 32(19), 3047-3048. https://doi.org/10.1093/bioinformatics/btw354

Funkhouser, L., \& Bordenstein, S. (2013). Mom Knows Best: The Universality of Maternal Microbial Transmission. PLoS Biology, 11(8), e1001631. https://doi.org/10.1371/journal.pbio.1001631

Gutierrez-Roman, M. I., Holguin-Melendez, F., Dunn, M. F., Guillen-Navarro, K., \& Huerta-Palacios, G. (2015). Antifungal activity of Serratia marcescens CFFSUR-B2 purified chitinolytic enzymes and prodigiosin 
against Mycosphaerella fijiensis, causal agent of black Sigatoka in banana (Musa spp.). BioControl, 60(4), 565-572. https://doi.org/10.1007/s10526-015-9655-6

Hird, S. M. (2017). Evolutionary biology needs wild microbiomes. Frontiers in Microbiology, 8. https://doi.org/10.3389/fmicb.2017.00725

Hong, P.-Y., Wheeler, E., Cann, I. K. O., \& Mackie, R. I. (2011). Phylogenetic analysis of the fecal microbial community in herbivorous land and marine iguanas of the Galapagos Islands using 16S rRNAbased pyrosequencing. The ISME Journal, 5(9), 1461-1470. https://doi.org/10.1038/ismej.2011.33

Jiang, H.-Y., Ma, J.-E., Li, J., Zhang, X.-J., Li, L.-M., He, N., Liu, H.-Y., Luo, S.-Y., Wu, Z.-J., Han, R.-C., \& Chen, J.-P. (2017). Diets alter the gut microbiome of crocodile lizards. Frontiers in Microbiology,8, 2073. https://doi.org/10.3389/fmicb.2017.02073

Kalbe, C., Marten, P., \& Berg, G. (1996). Strains of the genus Serratia as beneficial rhizobacteria of oilseed rape with antifungal properties. Microbiological Research, 151(4), 433-439. https://doi.org/10.1016/S09445013(96)80014-0

Keenan, S. W., Engel, A. S., \& Elsey, R. M. (2013). The alligator gut microbiome and implications for archosaur symbioses. Scientific Reports,3(1), 2877. https://doi.org/10.1038/srep02877

Kembel, S. W., Cowan, P. D., Helmus, M. R., Cornwell, W. K., Morlon, H., Ackerly, D. D., Blomberg, S. P., \& Webb, C. O. (2010). Picante: R tools for integrating phylogenies and ecology. Bioinformatics, 26 (11), 1463-1464. https://doi.org/10.1093/bioinformatics/btq166

Kohl, K. D., Brun, A., Magallanes, M., Brinkerhoff, J., Laspiur, A., Acosta, J. C., Caviedes-Vidal, E., \& Bordenstein, S. R. (2017). Gut microbial ecology of lizards: Insights into diversity in the wild, effects of captivity, variation across gut regions and transmission. Molecular Ecology,26(4), 1175-1189. https://doi.org/10.1111/mec.13921

Kreisinger, J., Čížková, D., Kropáčková, L., \& Albrecht, T. (2015). Cloacal microbiome structure in a long-distance migratory bird assessed using deep 16sRNA pyrosequencing. PloS One, 10(9), e0137401. https://doi.org/10.1371/journal.pone.0137401

Lee, W. Y. (2015). Avian gut microbiota and behavioral studies. Korean Journal of Ornithology. 22, 1-11.

Ley, R. E., Lozupone, C. A., Hamady, M., Knight, R., \& Gordon, J. I. (2008). Worlds within worlds: Evolution of the vertebrate gut microbiota. Nature Reviews Microbiology, 6(10), 776-788. https://doi.org/10.1038/nrmicro1978

Martin, B., Witten, D., \& Willis, A. (2020). corncob: Count Regression for Correlated Observations with the Beta-binomial (0.1.0) [R]. https://github.com/bryandmartin/corncob

Martin, M., Gilman, F. R., \& Weiss, S. L. (2010). Sex-specific asymmetry within the cloacal microbiota of the striped plateau lizard, Sceloporus virgatus.Symbiosis, 51(1), 97-105. https://doi.org/10.1007/s13199010-0078-y

McFall-Ngai, M., Hadfield, M. G., Bosch, T. C. G., Carey, H. V., Domazet-Lošo, T., Douglas, A. E., Dubilier, N., Eberl, G., Fukami, T., Gilbert, S. F., Hentschel, U., King, N., Kjelleberg, S., Knoll, A. H., Kremer, N., Mazmanian, S. K., Metcalf, J. L., Nealson, K., Pierce, N. E., .. Wernegreen, J. J. (2013). Animals in a bacterial world, a new imperative for the life sciences. Proceedings of the National Academy of Sciences, 110(9), 3229-3236. https://doi.org/10.1073/pnas.1218525110

McMurdie, P., \& Holmes, S. (2013). phyloseq: An R package for reproducible interactive analysis and graphics of microbiome census data. PLoS ONE,8(4), e61217. https://doi.org/10.1371/journal.pone.0061217

Moran, N, McCutcheon, J, \& Nakanachi, A. (2008). Genomes and evolution of heritable bacterial symbionts. Annual Review Genetics, 42, 165-190. 
Nyholm, S. V., \& McFall-Ngai, M. (2004). The winnowing: Establishing the squid-vibrio symbiosis. Nature Reviews Microbiology, 2(8), 632-642. https://doi.org/10.1038/nrmicro957

Oksanen, J., Blanchet, F. G., Friendly, M., Kindt, R., Legendre, P., McGlinn, D., Minchin, P. R., O'Hara, R., Simpson, G. L., Solymos, P., Stevens, M. H. H., Szoecs, E., \& Wagner, H. (2019). vegan: Community Ecology Package(2.5-6) [Computer software]. https://CRAN.R-project.org/package=vegan

Quast, C., Pruesse, E., Yilmaz, P., Gerken, J., Schweer, T., Yarza, P., Peplies, J., \& Glöckner, F. O. (2013). The SILVA ribosomal RNA gene database project: Improved data processing and web-based tools. Nucleic Acids Research, 41(D1), D590-D596. https://doi.org/10.1093/nar/gks1219

R Core Team. (2020).R: A language and environment for statistical computing. R Foundation for Statistical Computing. https://www.R-project.org/

Reese, A. T., \& Dunn, R. R. (2018). Drivers of microbiome biodiversity: A review of general rules, feces, and ignorance. MBio, 9(4). https://doi.org/10.1128/mBio.01294-18

Rose, B. (1981). Factors Affecting Activity in Sceloporus Virgatus. Ecology,62(3), 706-716. https://doi.org/10.2307/1937739

Sarmiento, C., Zalamea, P.-C., Dalling, J. W., Davis, A. S., Stump, S. M., U'Ren, J. M., \& Arnold, A. E. (2017). Soilborne fungi have host affinity and host-specific effects on seed germination and survival in a lowland tropical forest. Proceedings of the National Academy of Sciences,114(43), 11458-11463. https://doi.org/10.1073/pnas.1706324114

Schliep, K., Potts, A. A., Morrison, D. A., \& Grimm, G. W. (2016). Intertwining phylogenetic trees and networks [Preprint]. PeerJ Preprints. https://doi.org/10.7287/peerj.preprints.2054v1

Shterzer, N., Rothschild, N., Sbehat, Y., Stern, E., Nazarov, A., \& Mills, E. (2020). Large overlap between the intestinal and reproductive tract microbiomes of chickens. Frontiers in Microbiology, 11. https://doi.org/10.3389/fmicb.2020.01508

Stanley, D., Geier, M. S., Chen, H., Hughes, R. J., \& Moore, R. J. (2015). Comparison of fecal and cecal microbiotas reveals qualitative similarities but quantitative differences. BMC Microbiology, 15, 1-11.

Stebbins, R. C. (2003). A Peterson Field Guide to Western Reptiles and AmphibiansR. T. Peterson, Ed.; 3rd edition. Houghton Mifflin Harcourt.

Taylor, M. J., Mannan, R. W., U'Ren, J. M., Garber, N. P., Gallery, R. E., \& Arnold, A. E. (2019). Age-related variation in the oral microbiome of urban Cooper's hawks (Accipiter cooperii). BMC Microbiology,19(1), 47. https://doi.org/10.1186/s12866-019-1413-y

Trevelline, B. K., Fontaine, S. S., Hartup, B. K., \& Kohl, K. D. (2019). Conservation biology needs a microbial renaissance: A call for the consideration of host-associated microbiota in wildlife management practices.Proceedings of the Royal Society B: Biological Sciences,286(1895), 20182448. https://doi.org/10.1098/rspb.2018.2448

Videvall, E., Strandh, M., Engelbrecht, A., Cloete, S., \& Cornwallis, C. K. (2018). Measuring the gut microbiome in birds: Comparison of faecal and cloacal sampling. Molecular Ecology Resources, 18(3), 424-434. https://doi.org/10.1111/1755-0998.12744

Vinegar, M. (1975). Demography of the striped plateau lizard, Sceloporus virgatus.Ecology, 56(1), 172-182.

Wen, C., Li, Q., Lan, F., Li, X., Li, G., Yan, Y., Wu, G., Yang, N., \& Sun, C. (2021). Microbiota continuum along the chicken oviduct and its association with host genetics and egg formation. Poultry Science, 101104. https://doi.org/10.1016/j.psj.2021.101104 
White, J., Richard, M., Massot, M., \& Meylan, S. (2011). Cloacal bacterial diversity increases with multiple mates: Evidence of sexual transmission in female common lizards. PLOS ONE, 6(7), e22339. https://doi.org/10.1371/journal.pone.0022339

Wickham, H. (2017). ggplot2-Elegant Graphics for Data Analysis (2nd Edition). Journal of Statistical Software, 77 .

Williams, T., \& Athrey, G. (2020). Cloacal Swabs Are Unreliable Sources for Estimating Lower GastroIntestinal Tract Microbiota Membership and Structure in Broiler Chickens. Microorganisms, 8(5), 718. https://doi.org/10.3390/microorganisms 8050718

Wright, E., S. (2016). Using DECIPHER v2.0 to Analyze Big Biological Sequence Data in R. The R Journal, 8(1), 352. https://doi.org/10.32614/RJ-2016-025

Yasuda, K., Oh, K., Ren, B., Tickle, T. L., Franzosa, E. A., Wachtman, L. M., Miller, A. D., Westmoreland, S. V., Mansfield, K. G., Vallender, E. J., Miller, G. M., Rowlett, J. K., Gevers, D., Huttenhower, C., \& Morgan, X. C. (2015). Biogeography of the intestinal mucosal and lumenal microbiome in the rhesus macaque. Cell Host 83 Microbe, 17(3), 385-391. https://doi.org/10.1016/j.chom.2015.01.015

Zhang, C., Derrien, M., Levenez, F., Brazeilles, R., Ballal, S., Kim, J., Degivry, M.-C., Quéré, G., Garault, P., van Hylckama Vlieg, J. E. T., Garrett, W. S., Doré, J., \& Veiga, P. (2016). Ecological robustness of the gut microbiota in response to ingestion of transient food-borne microbes. The ISME Journal, 10(9), 2235-2245. https://doi.org/10.1038/ismej.2016.13

\section{Data Availability}

The sequences and associated metadata used to support the conclusions of this study are available in the sequence read archive of NCBI (BioProject PRJNA687551). R scripts and csv files are available from the corresponding author on request.

\section{Author Contributions}

All authors contributed to the study conception. Marie E. Bunker collected samples, performed all lab work, and drafted the manuscript. Stacey L. Weiss assisted with field work and sample collection. All authors edited drafts and approved of the final manuscript.

\section{Figure Legends}

Fig. 1 Mean (A) Shannon diversity (B)richness and (C) Faith's phylogenetic diversity of femaleS. virgatus cloacal swabs and tissue types. Although cloacal tissue diversity was lower than the other tissue types and the swabs, this difference was not statistically significant in any measure. Error bars represent standard error

Fig. 2 Beta diversity of $S$. virgatus cloacal swabs and tissue types. (A) Non-metric multi-dimensional scaling (NMDS) plot created by using Bray Curtis distance to calculate pairwise distances based on community composition; 3 dimensions were used to calculate distances but only the most influential two are pictured here. Samples clustered using (B) weighted and (C) unweighted UniFrac distances were used to create Principal Coordinates Analysis (PCoA) plots. Hulls and ellipses are colored by sample type

Fig. 3 Percent composition of bacterial families in cloacal swabs and tissue types. Each vertical bar represents one sample. Colored portions of the bars represent the relative abundance of the top ten most abundant taxa; the remaining taxa were combined into the "other" category. The y-axis indicates percent composition of total reads for that sample. Raw data are provided in Online Resource 1

Fig. 4 Effect of defecation on cloacal swab microbiome samples relative to the fecal pellet microbiome. (A) Shannon diversity index values, (B) richness, and (C) Faith's phylogenetic diversity of S. virgatus cloacal swabs before and after defecation, and fecal pellets. The fecal pellets have significantly higher diversity than the pre-defecation swabs in all metrics, but there were no differences between pre- and post-defecation swabs. Error bars represent standard error 
Fig. 5 Beta diversity of $S$. virgatus cloacal swabs and feces. (A) Non-metric multi-dimensional scaling (NMDS) plots created by using Bray Curtis distance to calculate pairwise distances based on community composition of cloacal swabs of $S$. virgatuslizards before and after defecation, as well as fecal samples from the same individuals. 2 dimensions were used to calculate distances. Samples clustered using (B) weighted and (C) unweighted UniFrac distances to create Principal Coordinates Analysis (PCoA) plots. Hulls and ellipses are colored by sample type

Fig. 6. Percent composition of bacterial families in pre- and post-defecation swabs, and fecal samples from S. virgatusindividuals. Each vertical bar represents one sample. Colored portions of the bars represent the relative abundance of the top ten most abundant taxa; the remaining taxa were combined into the "other" category. The y-axis indicates the percent composition of total reads for that sample. Raw data are provided in Online Resource 1

Fig. 7. Percent composition of the bacterial phyla known to dominate the vertebrate gut microbiome found in all S. virgatussamples from Study 1 and Study 2. Each vertical bar represents the mean percent composition for a given sample type. Raw data are provided in Online Resource 2
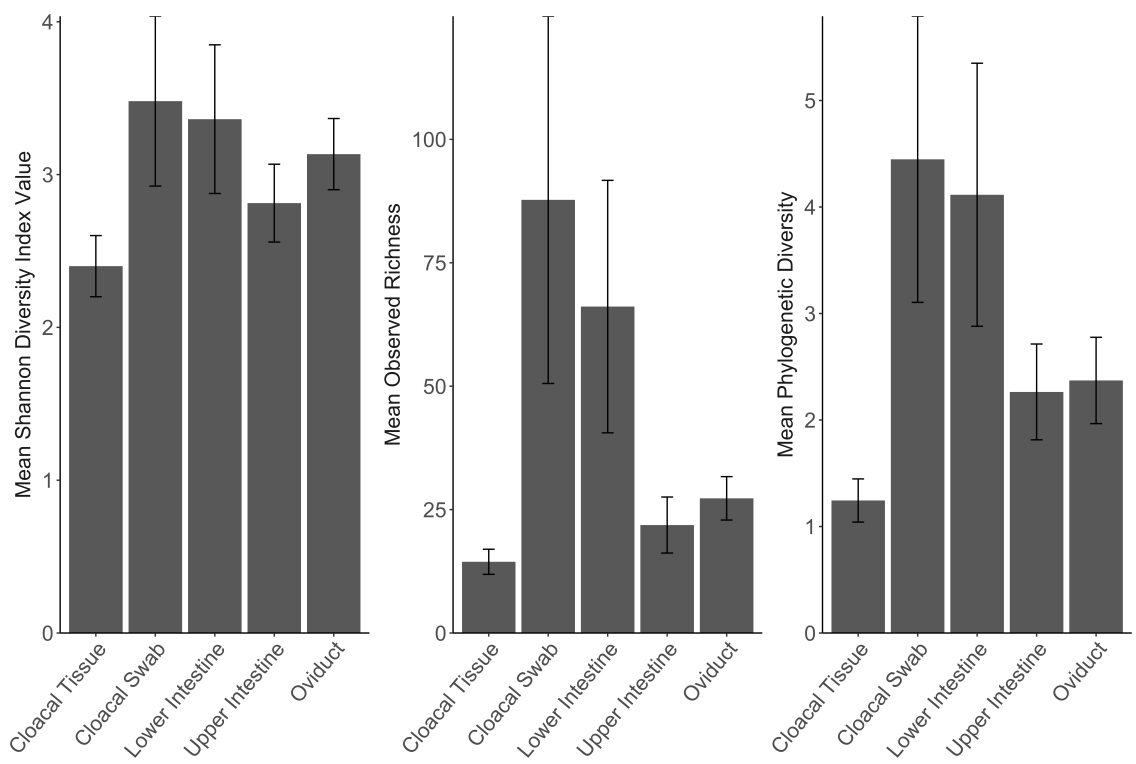


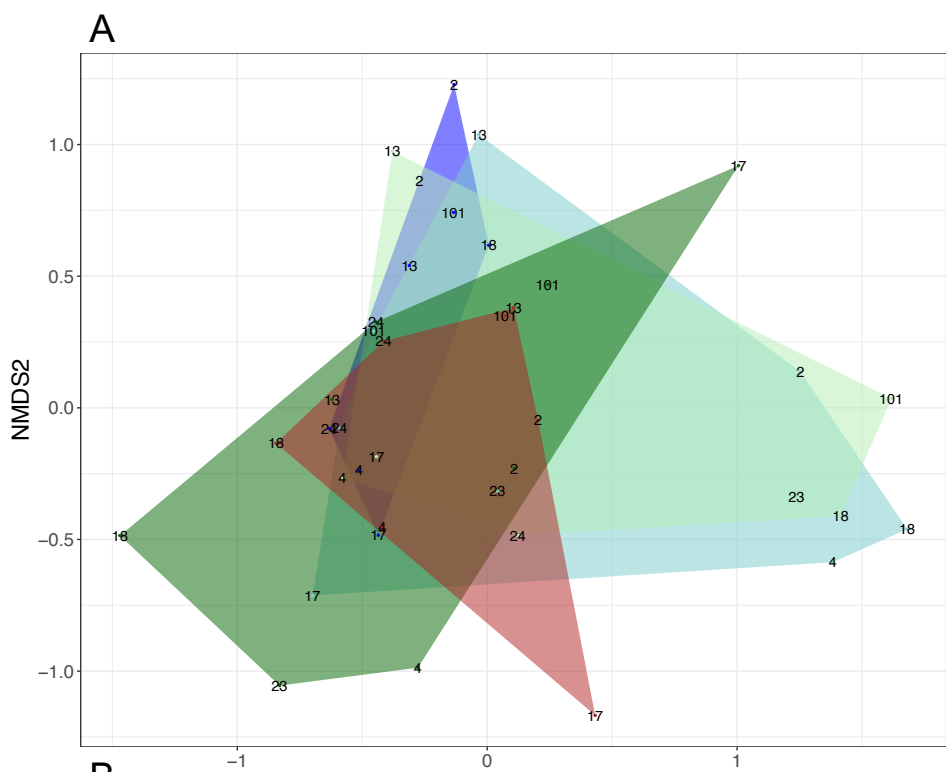

Tissue Type

- Cloacal Tissue Cloacal Swab Lower Intestine Upper Intestine - Oviduct

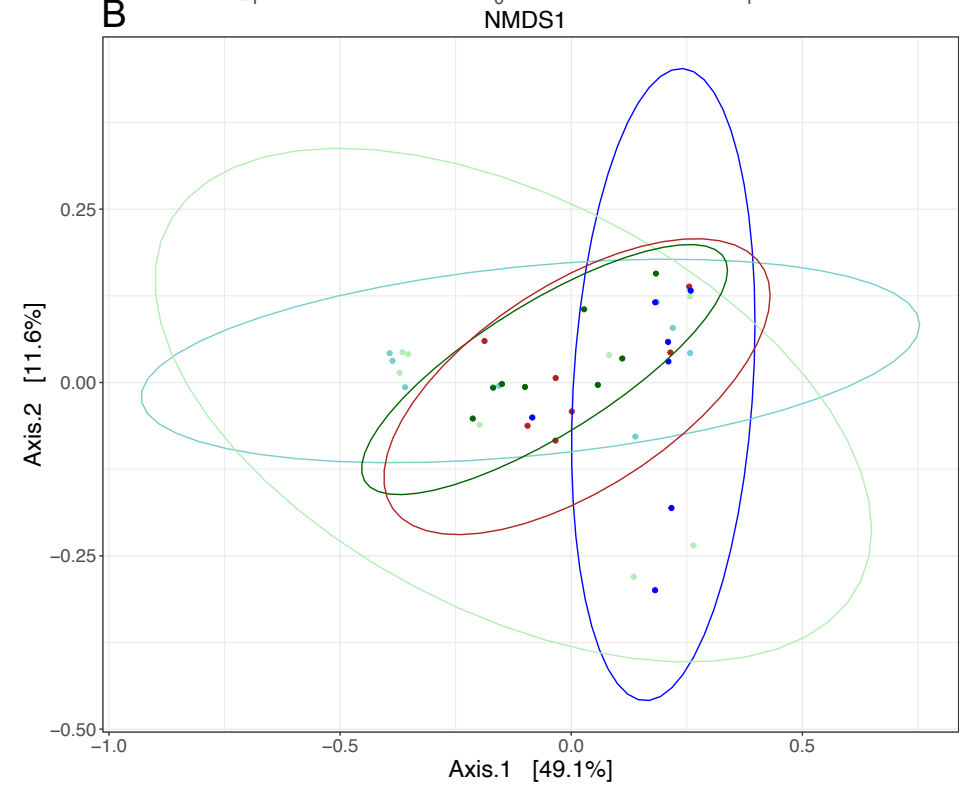

Tissue Type

- Cloacal Tissue

Cloacal Swab

Lower Intestine

- Upper Intestin

$$
\text { - Opiduat }
$$

C

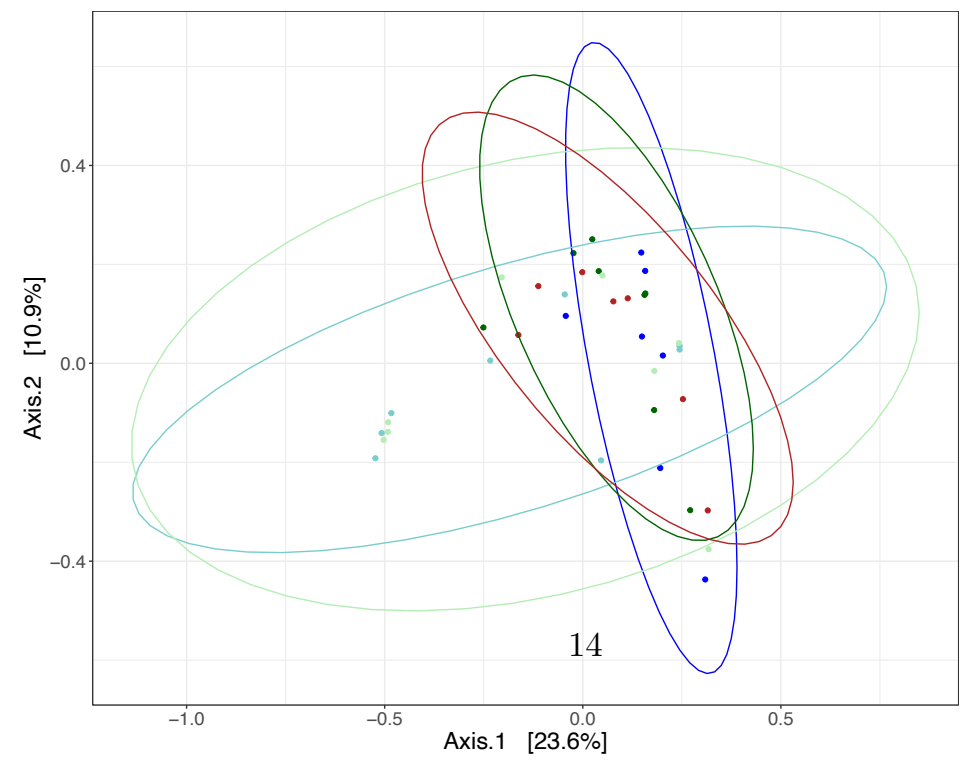

Tissue Type

- Cloacal Tissue Lower Intestine

- Upper Intestine

- Oviduct 

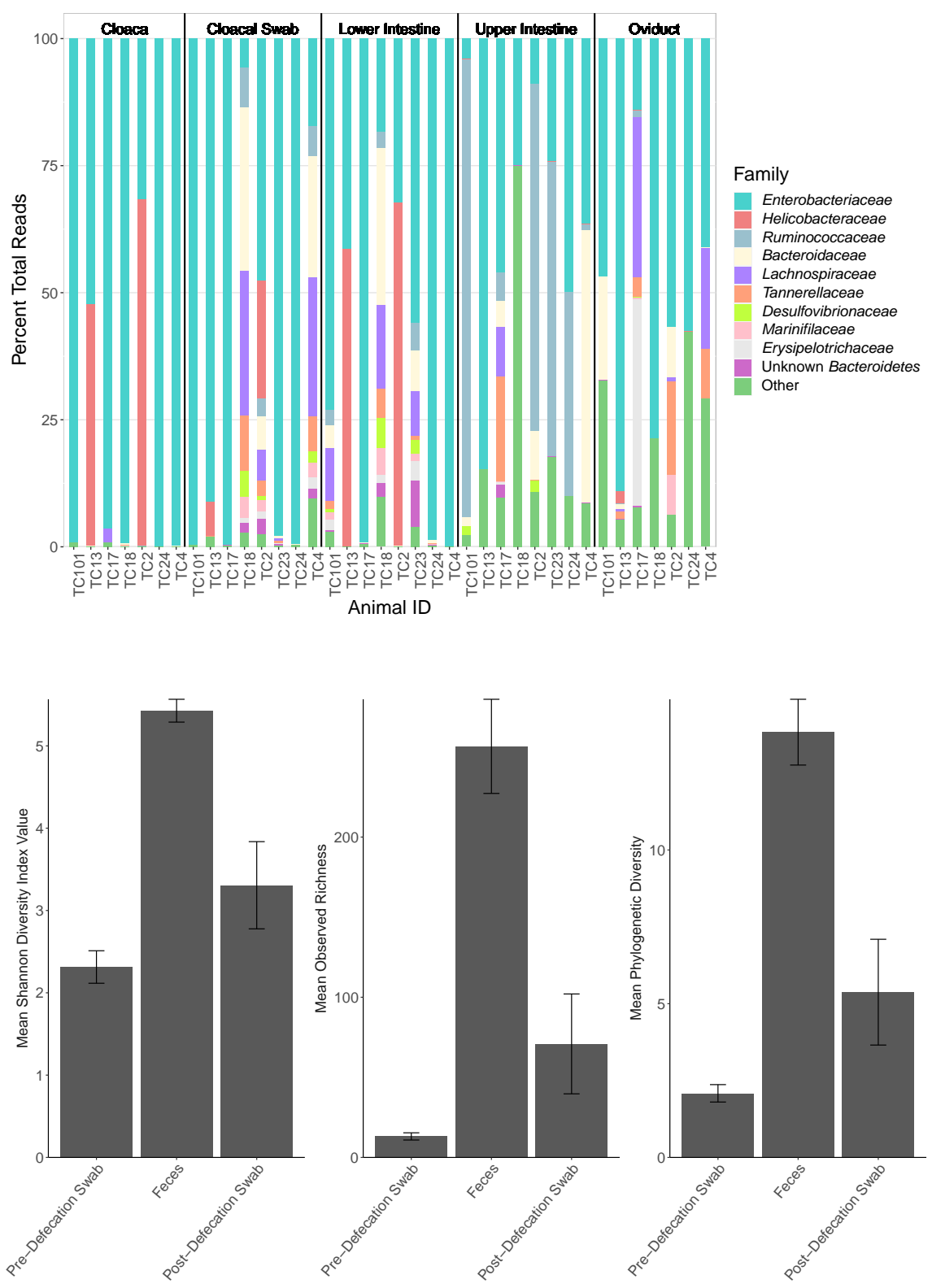


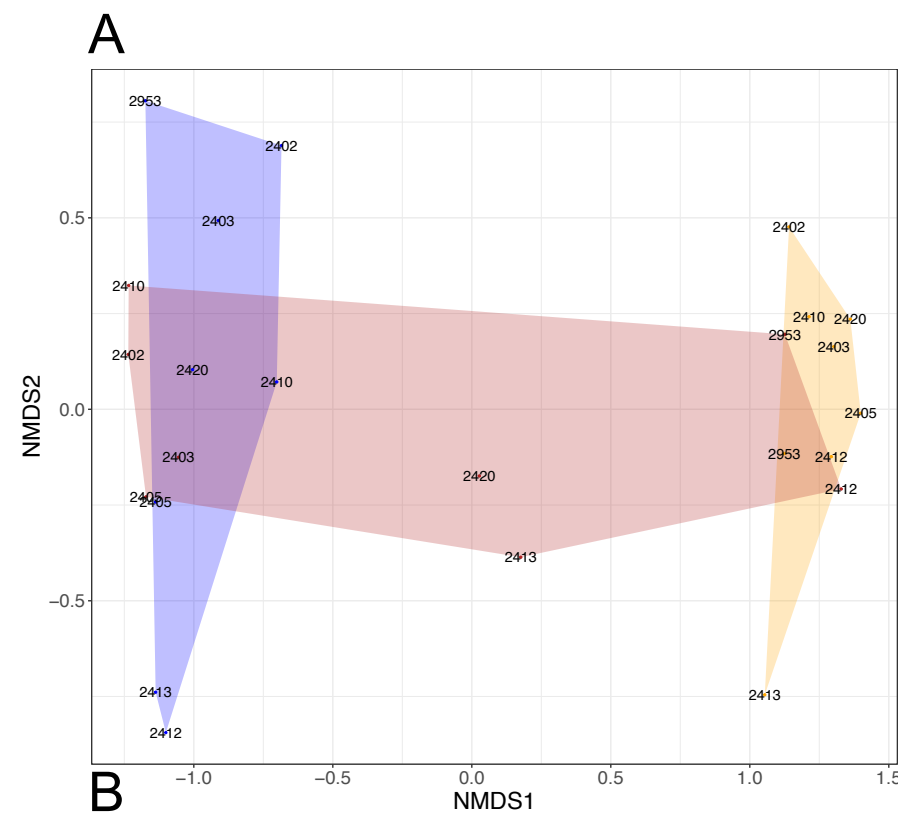

Sample Type

- Pre-Defecation Swab

- Post-Defecation Swab

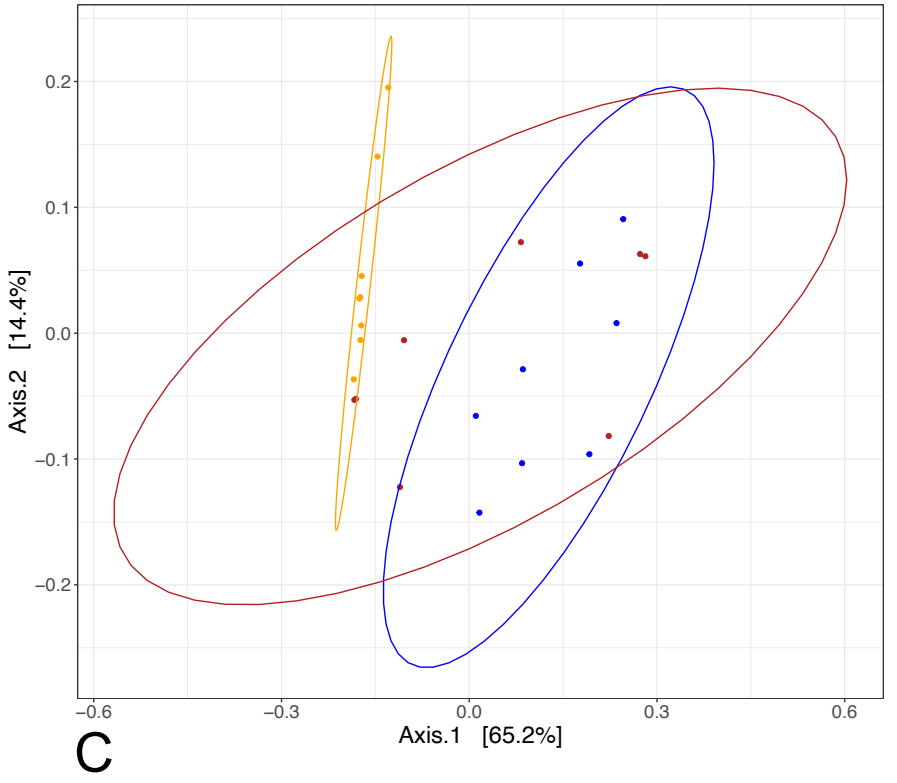

Sample Type

- Pre-Defecation Swab

- Feces

Post-Defecation Swab

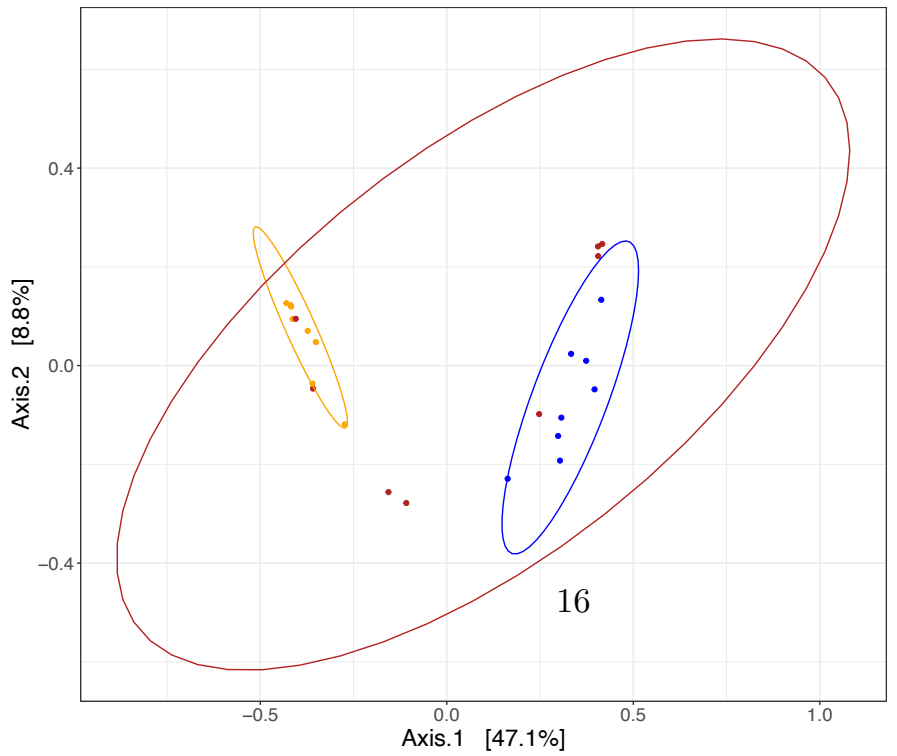

Sample Type

- Pre-Defecation Swab

$\rightarrow$ Feces

- Post-Defecation Swab 

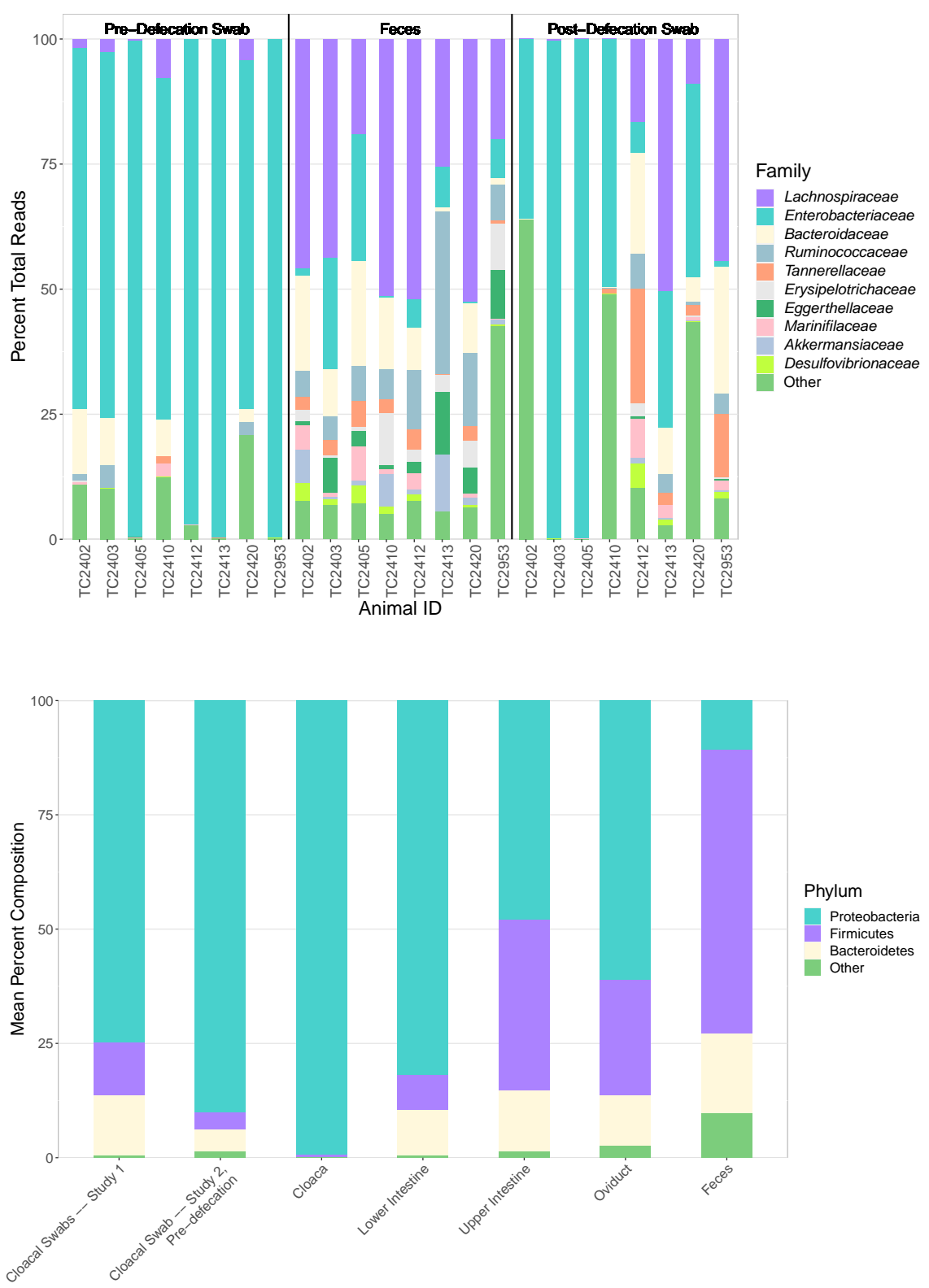